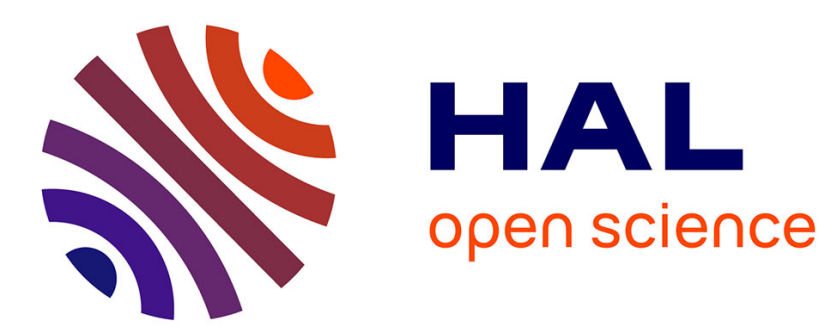

\title{
A polarization-based fast numerical method for computing the effective conductivity of composites
}

\author{
Vincent Monchiet, Guy Bonnet
}

\section{To cite this version:}

Vincent Monchiet, Guy Bonnet. A polarization-based fast numerical method for computing the effective conductivity of composites. International Journal of Numerical Methods for Heat and Fluid Flow, 2013, 23 (7), pp.1256-1271. hal-01078486

\section{HAL Id: hal-01078486 https://hal.science/hal-01078486}

Submitted on 15 Mar 2016

HAL is a multi-disciplinary open access archive for the deposit and dissemination of scientific research documents, whether they are published or not. The documents may come from teaching and research institutions in France or abroad, or from public or private research centers.
L'archive ouverte pluridisciplinaire HAL, est destinée au dépôt et à la diffusion de documents scientifiques de niveau recherche, publiés ou non, émanant des établissements d'enseignement et de recherche français ou étrangers, des laboratoires publics ou privés. 


\title{
A polarization-based fast numerical method for computing the effective conductivity of composites
}

\author{
Vincent Monchiet and Guy Bonnet \\ Université Paris-Est, Laboratoire Modélisation et Simulation Multi Echelle, \\ Marne la Vallée Cedex, France
}

\begin{abstract}
Purpose - The paper deals with the development of an improved fast Fourier transform (FFT)-based numerical method for computing the effective properties of composite conductors. The convergence of the basic FFT-based methods is recognized to depend drastically on the contrast between the phases. For instance, the primal formulation is not suited for solving the problems with high conductivity whereas the dual formulation is computationally costly for problems with high resistivity. Consequently, it raises the problem of computing the properties of composites containing both highly conductive and resistive inchusions.

Design/methodology/approach - In the present work, the authors' propose a new iterative scheme for solving that kind of problems which is formulated in term of the polarization.

Findings - The capability and relevance of this iterative scheme is illustrated through numerical implementation in the case of composites containing squared inclusions. It is shown that the rate of convergence is increased and thus, particularly when the case of high contrasts is considered. The predominance of the polarization based iterative scheme (PBIS) over existing ones is also illustrated in the case of a composite containing both highly conductive and highly resistive inclusions.

Originality/value - The method is easy to implement and uses the same ingredients as the basic schemes the FFT and the exact expression of the Green tensor in the Fourier space. Moreover, its convergence conditions do not depend on the conductivity properties of the constituents, which then constitutes the main difference with other existing iterative schemes. The method can then be applied for computing the effective properties of composites conductors with arbitrary contrasts.
\end{abstract}

Keywords Composition conductors, Homogenization, Fast Fourier transform, Polarization, Fourier transforms, Heat conduction

Paper type Research paper

\section{Introduction}

A key problem, of considerable technological importance, is to determine effective properties of composites that are governing the behavior at the macroscopic scale. In addition to methods using specific inclusions shapes (Kim and Torquato, 1962; Lu and Lin, 1996; Mercier et al, 2000), standard numerical toolsare used for solving the problem posed over the unit cell of periodicity, as for example: the finite elements methods (Gusev, 1997; Myroshnychenko and Brosseau, 2005), the boundary element methods (Eischen and Torquato, 1993), combined integral equations and multipole methods (Greengard and Moura, 1994; Helsing, 1995; McPhedran and Movchan, 1994).

An alternative method has been proposed in the mid-1990s by Moulinec and Suquet (1994) for the computation of effective properties of linear elastic periodic composite. The local cell problem is solved by means of an iterative scheme which uses the 
periodic Green's tensor for the strain and exact images of the microstructures. Note that en extension of the method has been recently proposed for piezoelectric composites by Brenner (2009). This method has several advantages over other existing ones. The approach uses a regular grid which can be used when using modern tools for characterizing the microstructure (tomograph). The discrete Fourier transform and its inverse are computed with the fast Fourier transform (FFT) which significantly increases the performance of the method. Moreover, the memory needed for solving the problem is significantly reduced compared to other methods.

However, the convergence of the FFT based algorithm introduced in Moulinec and Suquet (1994) (called primal iterative scheme (PIS) in the present paper) is known to be very sensitive with the contrast between the phases. More precisely, the rate of convergence decreases when the conductivity of the inclusions increases. Moreover, the case of infinitely conductive inclusions cannot be handled by the method since the algorithm diverges. A dual formulation of the original iterative scheme (Bhattacharya and Suquet, 2005; Bonnet, 2007) is better suited in the domain of high conductivity. However, it is computationally costly in the domain of low conductivity. Tocircumvent the incapacity of the original approach (Moulinec and Suquet, 1994), other iterative schemes have been developed during the ten past years. For instance, the "accelerated" scheme has been proposed by Eyre and Milton (1999), however, the method still diverges for infinite conductivities. To address the problems of composites with infinite contrast, alternative methods, based on the minimization of augmented Lagrangians, have been proposed by Michel et al. (2000) and Michel et al. (2001). More recently, Brisard and Dormieux (2010) have proposed a new method based on the Hashin-Shtrikman variational principle (Hashin and Shtrikman, 1962; Willis, 1977), it uses a modified expression of the Green operator and the conjugate gradient method for the minimization.

In the present paper, we propose a simple iterative scheme, based on the polarization, which uses the same ingredients as the basic schemes and does not need sophisticated numerical tools. This method, presented here for composite conductors, presents the advantage to converge independently of the choice of the conductivities of the constituents. The paper contains three parts. In Section 2, we briefly recall the equations of the unit cell problem and of the inclusion problems in the context of the thermal conduction of periodic composites. In Section 3, we present the basic schemes (the primal and the dual). The rate of convergence and the limitations of these methods are discussed and illustrated through a few numerical calculations. In the last part of the paper (Section 4), we present the new polarization-based method. It is demonstrated that the convergence of this new iterative scheme is independent of the local properties. The relevance of this polarization-based algorithm over the basic schemes is shown through several applications with extremal conductivities.

Notations. $\underline{a}, \underline{A}$ : vectors; $\boldsymbol{a}, \boldsymbol{A}$ : two order tensors. $\underline{a} \cdot \underline{b}=a_{i} b_{i},(\boldsymbol{A} \cdot \underline{a})_{i}=A_{i j} a_{j}$, $A: B=A_{i j} B_{j i}$ where the summation convention on repeated indices is applied.

\section{Statement of the problem}

\subsection{The unit cell problem}

A periodic composite material is defined by a paralepipedic unit cell and three vectors defining the translations which leave the medium invariant. The volume of this cell is denoted by $V$, while $V_{\alpha}$ for $\alpha=1, \ldots, N$ denotes the volume of each constituent. 
In the present paper, we look for the determination of the periodic fields $\underline{e}(\underline{x})$ and $\underline{j}(\underline{x})$ solutions of the linear problem:

$$
\left\{\begin{array}{l}
\underline{e}(\underline{x})=\nabla \varphi(\underline{x}), \quad \forall \underline{x} \in V \\
\underline{j}(\underline{x})=K(\underline{x}) \cdot \underline{e}(\underline{x}), \quad \forall \underline{x} \in V \\
\operatorname{div}(\underline{j}(\underline{x})=0, \quad \forall \underline{x} \in V \\
\varphi(\underline{x})-\underline{E} \cdot \underline{x} \text { periodic } \\
\underline{j(x)} \cdot \underline{n} \text { antiperiodic }
\end{array}\right.
$$

for a prescribed mean value $\underline{E}=\langle\underline{e}(\underline{x})\rangle_{V}$ or $\underline{J}=\langle\underline{j}(\underline{x})\rangle_{V}$. Here, as elsewhere, the angular brackets, $\langle\cdot\rangle_{V}$, will be used to denote the volume averages over the unit cell of periodicity. Once this solution will be known, the relation giving $J$ as a function of $\underline{E}$ is used to define the homogenized properties, $\boldsymbol{K}^{\text {ham }}$. The above problem can describe various phenomena such as the thermal conduction, the magnetism or electricity of composite materials. For the thermal conduction problem, the pair $(\underline{e}, j)$ represents the gradient of temperature and the flux. In equation (1), $K(x)$ is the conductivity and the resistivity is denoted by $\boldsymbol{R}(\underline{x})$. They can be put into the form:

$$
\begin{gathered}
\boldsymbol{K}(\underline{x})=\sum_{\alpha} I_{\alpha}(\underline{x}) \boldsymbol{K}_{\alpha}, \quad \boldsymbol{R}(\underline{x})=\sum_{\alpha} I_{\alpha}(\underline{x}) \boldsymbol{R}_{\alpha} \\
\text { with : } \quad I_{\alpha}(\underline{x})= \begin{cases}1 & \text { if } \underline{x} \in V_{\alpha} \\
0 & \text { if } \underline{x} \in V-V_{\alpha}\end{cases}
\end{gathered}
$$

$I_{\alpha}(\underline{x})$ for $\alpha=1, \ldots, M$ are the characteristic functions describing volumes $V_{\alpha x}$. Due to the periodicity, it is convenient to expand the fields $\underline{e}(\underline{x})$ and $\underline{j}(\underline{x})$ along Fourier series:

$$
\left.\underline{e}(\underline{x})=\sum_{n} \underline{\underline{e}}\left(\underline{\xi}_{n}\right) \exp \left(i \underline{\underline{\xi}}_{n} \cdot \underline{x}\right) \quad \underline{j} \underline{x}\right)=\sum_{n} \underline{j}\left(\underline{\xi}_{n}\right) \exp \left(i \underline{\xi}_{n} \cdot \underline{x}\right)
$$

where the $\xi_{n}$ are the discrete wave vectors arranged along a discrete network having a period $2 \pi / b_{i}$ in the direction $x_{i}\left(b_{i}\right.$ denotes the half width of the parallepipedic unit cell). In equation $(3), \underline{e}(\xi)$ and $\underline{j}(\xi)$ denote the Fourier transforms of $\underline{e}(\underline{x})$ and $\underline{j}(\underline{x})$ :

$$
\begin{array}{ll}
\underline{e}(\underline{\xi})=<\underline{e}(\underline{x}) \exp (-i \underline{\xi} \cdot \underline{x})>_{V} & \text { with }: \underline{e}(\underline{\xi}=0)=\underline{E} \\
\underline{j}(\underline{\xi})=<\underline{j}(\underline{x}) \exp (-i \underline{\xi} \cdot \underline{x})>_{V} & \text { with }: \underline{j} \underline{\xi}=0)=\underline{J}
\end{array}
$$

The difference between quantities in Fourier space and in real space is denoted by adding the variable, for example $\underline{e}(\underline{\xi})$ for the Fourier transform of $\underline{e}(\underline{x})$ with the same symbol " $\underline{e}$ " in both cases.

In the real space, the local variables $\underline{\underline{e}}(\underline{x})$ and $j(\underline{x})$ are, respectively, rotational and divergence free vectors, which leads, in Fourier space, to:

$$
\forall \underline{\xi} \neq 0:\left\{\begin{array}{l}
\boldsymbol{Q}(\underline{\xi}) \cdot \underline{e}(\underline{\xi})=0 \\
P(\underline{\xi}) \cdot \underline{j} \underline{\xi})=0
\end{array}\right.
$$


where $\boldsymbol{Q}(\xi)$ and $\boldsymbol{P}(\underline{\xi})$ are given by:

$$
Q(\underline{\xi})=I-P(\underline{\xi}), \quad P(\underline{\xi})=\frac{1}{\|\underline{\xi}\|^{2}} \underline{\xi} \otimes \underline{\xi}
$$

where $\boldsymbol{P}(\xi)$ and $\boldsymbol{Q}(\xi)$ are the two idempotent and orthogonal projectors:

$$
\forall \underline{\xi} \neq 0:\left\{\begin{array}{l}
P(\underline{\xi}) \cdot P(\underline{\xi})=P(\underline{\xi}) \\
Q(\underline{\xi}) \cdot Q(\underline{\xi})=Q(\underline{\xi}) \\
P(\underline{\xi}) \cdot Q(\underline{\xi})=Q(\underline{\xi}) \cdot B(\underline{\xi})=0 \\
I=P(\underline{\xi})+Q(\underline{\xi})
\end{array}\right.
$$

The action of $\boldsymbol{Q}(\xi)$ on a non-uniform vector $\underline{a}(\xi)$ gives the projection of $\underline{a}(\xi)$ along the plane of normal $\bar{\xi}$ denoted $\mathcal{Q}_{\xi}$. The action of $\bar{P}(\bar{\xi})$ on a non-uniform vector $\underline{a}(\xi)$ provides the "out of plane" components, namely along ${ }^{2} \mathcal{P}_{\xi}$ the supplementary of $\bar{Q}_{\xi}$. The two orthogonal subspaces $\mathcal{P}_{\xi}$ and $\mathcal{Q}_{\xi}$ have, respectively, dimensions 1 and 2 for three dimensional problems.

\subsection{A related problem}

Consider in this section a fictitious infinite homogeneous medium having a conductivity $K^{0}$. This medium is submitted to a periodic polarization $p(x)$ and to an applied uniform gradient of temperature $\underline{E}$. Since the fields $\underline{\underline{e}} \underline{x}$ ) and $\underline{j} \underline{x}$ are related by:

$$
\underline{j}(\underline{x})=K^{0} \cdot \underline{e}(\underline{x})+\underline{p}(\underline{x})
$$

the computation of the solution for $\underline{e}(\underline{x})$ and $(j(x))$ is possible because the problem becomes algebraic in the Fourier space. For instance, the gradient of temperature reads:

$$
\begin{cases}\underline{e}(\underline{\xi})=-\Gamma^{0}(\underline{\xi}) \cdot \underline{p(\xi)} & \forall \underline{\xi} \neq 0 \\ \underline{e}(\underline{\xi})=\underline{E} & \text { for }: \underline{\xi}=0\end{cases}
$$

In this equation, the Green's tensor, $\Gamma^{0}(\xi)$, reads:

$$
\forall \underline{\xi} \neq 0: \Gamma^{0}(\underline{\xi})=\frac{1}{K_{0}: P(\underline{\xi})} P(\underline{\xi})
$$

and $\Gamma^{0}(\xi)=0$ when $\xi=0$. Note that $\Gamma^{0}\left(\underline{\xi}\right.$ has the properties, $\forall \underline{\xi} \neq 0, P(\xi) \cdot \Gamma^{0}(\xi)=$ $\Gamma^{0}(\xi)$ and $Q(\xi) \cdot \Gamma^{0}(\xi)=0$ which means that the vector, given by equation $(9)$ is rotational free. The flux, $\underline{j}(\underline{x})$, is given by:

$$
\begin{cases}j(\underline{\xi})=\Delta^{0}(\underline{\xi}) \cdot R^{0} \cdot \underline{p(\xi)} & \forall \underline{\xi} \neq 0 \\ j(\underline{\xi})=\underline{J} & \text { for }: \underline{\xi}=0\end{cases}
$$

where $\underline{I}=\boldsymbol{K}^{0} \cdot \underline{E}+\underline{P}$ and $\left.\underline{P}=\langle\underline{p} \underline{x})\right\rangle_{V}$. 
$\Delta^{0}(\xi)$ is the second Green tensor, defined in the Fourier space, by:

$$
\forall \underline{\xi} \neq 0: \Delta^{0}(\underline{\xi})=K^{0}-K^{0} \cdot \Gamma^{0}(\underline{\xi}) \cdot K^{0}
$$

and $\Delta^{0}(\xi)=0$ for $\xi=0$. Note that $\Delta^{0}(\underline{\xi})$ has the properties, $\forall \underline{\xi} \neq 0, P(\xi) \cdot \Delta^{0}(\xi)=0$ and $Q\left(\bar{\xi} \cdot \Delta^{0}(\xi)=\Delta^{0}(\xi)\right.$ which means that the vector, given by equation (11), is divergence free. When an isotropic reference medium is considered, $K^{0}=k_{0} I$, the Green tensors reduce to $\Gamma^{0}(\xi)=1 / k_{0} \boldsymbol{P}(\xi)$ and $\Delta^{0}(\xi)=k_{0} \boldsymbol{Q}(\xi)$.

3. The basic iterative scheme

3.1 The primal iterative scheme

By considering in equation (9), the following definition for the polarization: $\underline{p}(\underline{x})=\left(K(\underline{x})-K^{0}\right) \cdot \underline{e} \underline{x}$, the solution, $\left.\underline{e} \underline{x}\right)$ complies with the integral equation:

$$
\underline{e}(\underline{x})=\underline{E}-\Gamma^{0}(\underline{x}) *\left[\left(K(\underline{x})-K^{0}\right) \cdot \underline{e}(\underline{x})\right]
$$

A possible way to compute the solution of the above equation has been first proposed by Moulinec and Suquet (1994) for linear elastic composite, along the lines of a method which was first introduced for composite conductors by Brown (1955) and later by Kroener (1972). It consists in expanding the solution into Neumann series, each term being obtained by the following recurrence relation:

$$
\underline{e}^{i+1}(\underline{x})=\underline{E}-\Gamma^{0}(\underline{x}) *\left[\left(K(\underline{x})-K^{0}\right) \cdot \underline{j}(\underline{x})\right]
$$

which is initiated by: $\underline{e}^{1}(\underline{x})=\underline{E}$. A simplification of the above iterative scheme is possible. For this, it can be observed that $\Gamma^{0}(\underline{x}) *\left[K^{0} \cdot \underline{e}^{i}(\underline{x})\right]=\underline{e}(\underline{x})-\underline{E}$ which is true for any rotational free vector $\underline{\underline{e}}(\underline{x})$. To summarize, the iterative scheme becomes:

$$
\left\{\begin{array}{l}
\underline{f}(\underline{x})=K(\underline{x}) \cdot \underline{e}^{i}(\underline{x}) \\
\dot{f}(\underline{\xi})=\mathcal{F}(\underline{\dot{f}}(\underline{x})) \\
\text { convergence test } \\
\underline{\underline{j}}^{i+1}(\underline{\xi})=\underline{\dot{j}}(\underline{\xi})-\Gamma^{0}(\underline{\xi}) \cdot \underline{\dot{j}}(\underline{\xi}) \\
e^{i+1}(\underline{x})=\mathcal{F}^{-1}\left(\underline{e}^{j+1}(\underline{\xi})\right)
\end{array}\right.
$$

in which $\mathcal{F}$ and $\mathcal{F}^{-1}$ denote the Fourier transform and its inverse. Their computations are effected by means of the FFT algorithm which presents the advantage to greatly reduce the calculations times. Note that the flux, $j(\underline{x})$, is only divergence free at convergence of the iterative scheme. For this reason, the following convergence test is applied in equation (15):

$$
\frac{\|P(\underline{\xi}) \cdot \underline{j}(\underline{\xi})\|}{\| \underline{i}^{i}(\underline{\underline{\xi}} \|}<\varepsilon
$$

where $\|\bullet\|$ denotes the Frobenius norm. The value $\epsilon=10^{-3}$ has been used in this paper. 
The conditions giving the values of the conductivity of the reference medium producing the convergence of the iterative scheme are discussed in Appendix 1. It is shown that the convergence will be attained if:

$$
2 k_{0}>k(\underline{x}) \geq 0
$$

Note that the condition of convergence of the PIS has been already studied in Michel et al. (2001). However, in the present paper, we have obtained a minor modification of their initial result which conoern the particular case of cavities. In Michel et al. (2001), the authors investigate the conditions giving the convergence of the Neumann series. This series converges only if the eigenvalues of the operator are strictly comprised between -1 and 1 . By studying the eigenvalues of this operator which appears in the Neumann series, they found that the convergence cannot be ensured when the composite material comprises voids, because this case produces eigenvalues of the operator equal to 1 . In the present paper, we focus our attention on the convergence of the iterative scheme instead of the Neumann series. In that case, the eigenvalue 1 is allowed and we found that the converge occurs also for a composite with cavities. This result has been numerically recovered.

We now aim at investigating the rate of convergence of the primal scheme as a function of the contrast. To this end, we consider the case of the matrix/inclusions type composite conductor described on Figure 1. It is made up of long cylinders with a squared cross-section. By $a$, we denote the half width of the squares whereas the distance between the axis of two neighboring cylinders is denoted by $2 b$. In our applications we have chosen $a=b / 2$. Both phases are assumed to be isotropic and by $k_{M}$ we denote the conductivity of the matrix whereas the conductivity of the inclusions is denoted by $k_{r}$. The problem is two dimensional, a squared unit cell, as defined on Figure 1 (the dotted square), is then considered in our calculations.

The numerical integration of the algorithm is realized by replacing the exact Fourier transform by the discrete Fourier transform. The calculations are performed by considering 128 wave vectors. This number must be considered for obtaining a good

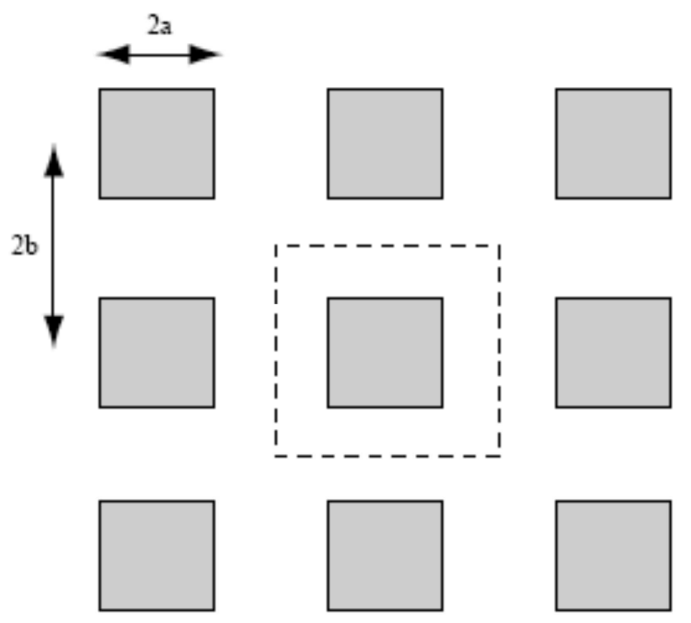

Figure 1. The unit cell of the periodic composite 
accuracy on the effective properties. Following Bonnet (2007), we use an exact representation of the conductivity, $K(\underline{x})$, in the Fourier space. In the original approach (Moulinec and Suquet, 1994), the local properties $K(\underline{x})$ are assigned at each point of a regular grid. Indeed, in many inclusion/matrix type problems, an exact expression for the Fourier transforms of the characteristic functions $I_{\alpha}(\underline{x})$, which appears in equation (2), can be obtained. For example, in the present case:

$$
\begin{aligned}
& \forall \underline{\xi} \neq 0: \quad\left\{\begin{array}{l}
I_{I}(\underline{\xi})=\frac{\sin \left(a \xi_{1}\right) \sin \left(a \xi_{2}\right)}{b^{2} \xi_{1} \xi_{2}} \\
I_{M}(\underline{\xi})=-I_{I}(\underline{\xi})
\end{array}\right. \\
& \forall \underline{\xi}=0: \quad I_{I}(\underline{\xi})=\frac{a^{2}}{b^{2}}, \quad I_{M}(\underline{\xi})=1-\frac{a^{2}}{b^{2}}
\end{aligned}
$$

In Bonnet (2007), it has been shown that the use of such "shape functions" improves the convergence of the iterative scheme. Following Milton (2002), we have chosen $k_{0}=\left(k_{I}+k_{M}\right) / 2$, which appears to be very close to the optimal reference (namely the one leading to the lower number of iterations at convergence). In Figure 2, we display the logarithm of the number of iterations $(\log (N))$ needed for having the convergence of the iterative scheme, as a function of the logarithm of the ratio $k_{7} / k_{M}$. It can be observed that in the range $\log \left(k_{I} / k_{M}\right) \leq 0$, the convergence of the iterative scheme is obtained quickly. When the conductivity of the inclusions is higher than the one of the matrix, namely in the range $\log \left(k_{I} / k_{M}\right) \geq 0$, the dependence between the number of iterations and the contrast $k_{I} / k_{M}$ is linear in a log-log frame. It suggests that this scheme is not suited for high conductivities since it becomes computationally costly. Moreover, the case of composites with infinite conductivity cannot be handled by the method.

Figure 2.

Diagram giving the

number of iterations at

convergence of the PS as

a function of the

ratio $k_{I} / k_{M}$

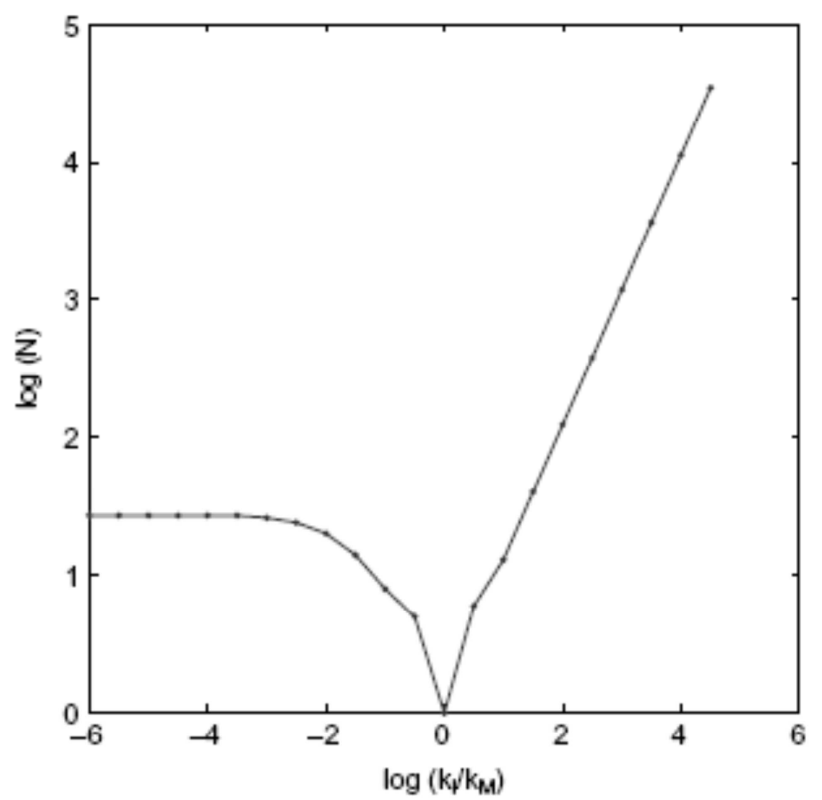


Note that the case of a cavity, corresponding to $k_{I}=0$, is not represented in Figure 2 since the contrast is comprised between $10^{-6}$ and $10^{6}$. However, the case of cavity has been tested and the convergence is attained after the same number of iterations as for $k_{I} / k_{M}=10^{-6}$.

\subsection{The dual iterative scheme}

A dual formulation of the iterative scheme is better suited when the inclusions of composites has a higher conductivity than the matrix. This dual formulation reads:

$$
\left\{\begin{array}{l}
\underline{\dot{e}}(\underline{x})=R(\underline{x}) \cdot \underline{j}^{i}(x) \\
\underline{\underline{e}}(\underline{\xi})=\mathcal{F}(\underline{\dot{e}}(\underline{x})) \\
\text { convergence test } \\
\underline{j}^{j+1}(\underline{\xi})=\underline{j}^{i}(\underline{\xi})-\Delta^{0}(\underline{\xi}) \cdot e^{i}(\underline{\xi}) \\
\underline{j}^{j+1}(\underline{x})=F^{-1}\left(\underline{j}^{+1}(\underline{\xi})\right.
\end{array}\right.
$$

which starts from the first term given by: $j^{1}(\underline{x})=J$. In equation (19), the flux $\underline{f}(\underline{x})$ is divergence free at each step of the iterative scheme while the gradient of temperature, $\underline{\underline{e}}(\underline{x})$, is rotational free only at convergence. Consequently, the following convergence test is used in equation (19):

$$
\frac{\left\|Q(\underline{\xi}) \cdot \underline{e}^{i}(\underline{\xi})\right\|}{\|\underline{e}(\underline{\xi})\|}<\epsilon
$$

In which the value $\epsilon=10^{-3}$ will be used in the following. The convergence of this second algorithm is ensured if (Appendix 2):

$$
\frac{2}{k_{0}}>\frac{1}{k(x)} \geq 0
$$

As for the PIS, we now aim at investigating the rate of convergence of the dual method. For this, we still consider the case of the composite defined on Figure 1. Dually to the first method, the resistivity of the reference medium defined by $1 / k_{0}=$ $\left(1 / k_{M}+1 / k_{I}\right) / 2$ has been considered On Figure 3, we represent the variations of the logarithm of the number of iterations with $\log \left(k_{I} / k_{M}\right)$. It can be observed that the convergence is quickly obtained for inclusions with high and infinite conductivity. However, this is now the domain of low conductivity for which the iterative scheme is computationally costly. Moreover, the case of inclusions with a null conductivity cannot be solved by using this second formulation.

In summary, from these two methods, the first one (the PIS) is better suited for computing the effective properties of composites with low conductive inclusions. In the other hand, the case of composites with low resistive inclusions must be solved by the dual formulation. However, when the composite contains both high and low conductive inclusions, the basic schemes appears to be inappropriate and the case of composites containing both inclusions leading to zero and infinite contrast cannot be handled with these numerical tools. 


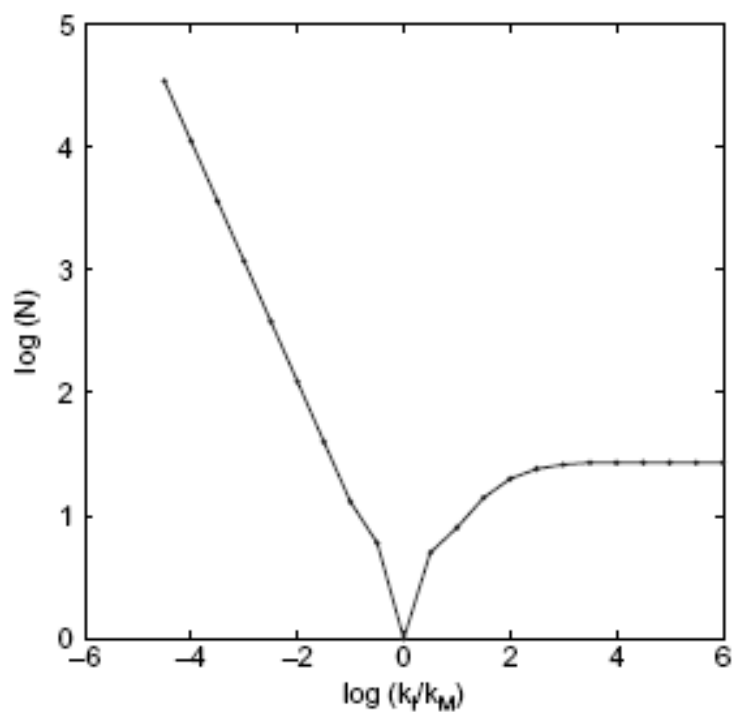

Figure 3.

Number of iterations

at convergence of the DIS as a function of the ratio $k_{I} / k_{M}$

\section{A polarization-based iterative scheme}

\subsection{The algorithm}

In this section, we propose a new approach for computing the effective properties of composites with arbitrary conductivities. It is based on the resolution of a fictitious problem in which a uniform polarization, $\underline{P}$ is prescribed to the unit cell instead of $\underline{E}$ or $J$. Due to the linearity of equations (1), there exist two linear relations giving the quantities $\underline{E}$ and $J$ as a function of $\underline{P}$.

$$
\underline{E}=A \cdot \underline{P}, \quad \underline{J}=B \cdot \underline{P}
$$

Since $J=\boldsymbol{K}^{0} \cdot \underline{\boldsymbol{E}}+\underline{P}$ the fourth order tensors $\boldsymbol{A}$ and $\boldsymbol{B}$ are linked by $\boldsymbol{B}=\boldsymbol{I}+\boldsymbol{K}^{0} \cdot \boldsymbol{A}$. The effective conductivity $\boldsymbol{K}^{\text {hom }}$ can be either expressed in term of tensor $\boldsymbol{A}$ or $\boldsymbol{B}$ :

$$
K^{\text {hom }}=A^{-1}+K^{0}=\left(I-B^{-1}\right)^{-1} \cdot K^{0}
$$

The solution of equation(1), with prescribed uniform polarization, can be computed by using the following iterative scheme:

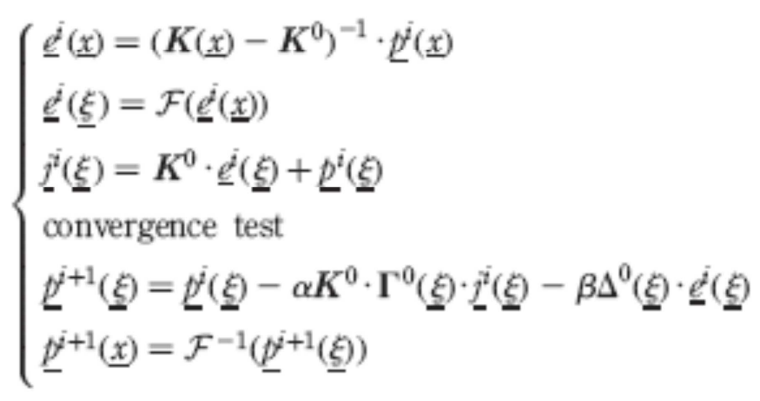


where $\underline{p}^{1}(\underline{x})=\underline{P}$ is taken as the first term of the series. In equation (24), $\alpha$ and $\beta$ are two coefficients which will be chosen therefore in order to obtain the convergence. When it is achieved, namely $p^{i+1}(\underline{x})=\underline{p}^{i}(\underline{x}), \underline{\underline{d}}(\xi)$ and $\dot{f}(\xi)$ are, respectively, a rotational and a divergence free vector. The iterative Scheme $(24)$ is then stopped when:

$$
\max \left(\frac{\left\|\boldsymbol{Q}(\underline{\xi}) \cdot \underline{e}^{i}(\underline{\xi})\right\|}{\| \underline{\underline{e}}) \|}, \frac{\left\|\boldsymbol{P}(\underline{\xi}) \cdot \underline{j}_{i}(\underline{\xi})\right\|}{\left\|\underline{j}_{i}(\underline{\xi})\right\|}\right)<\epsilon
$$

Again the value $\epsilon=10^{-3}$ is still considered in our applications.

Note that, as the basic schemes, the polarisation-based algorithm require one Fourier transform and one inverse Fourier Transform at each iteration. At convergence, one can compute $E, J$, the components of $\boldsymbol{A}$ and $\boldsymbol{B}$ and finally the homogenized properties of the composite with one of the two equations of equation (23).

\subsection{The conditions of convergence}

The convergence of an iterative scheme, having the general form $p^{i+1}=(I-L) \cdot p^{i}$, is ensured if all the eigenvalues of the operator $\boldsymbol{I}-\boldsymbol{L}$ are comprised in the interval ] - 1, 1]. Equivalently, the convergence is obtained if the eigenvalues of $L$ arecomprised in $[0,2[$. Based on this observation, we look for the eigenvalues of the operator:

$$
\alpha K^{0} \cdot \Gamma^{0}(\underline{x}) *\left(K(\underline{x}) \cdot \delta K^{-1}(\underline{x})\right)+\beta \Delta^{0}(\underline{x}) * \delta K^{-1}(\underline{x})
$$

where the following notation have been used: $\delta K(x)=K(x)-K^{0}$. Introducing an eigenvalue $\phi$ of that operator and its associated eigenvector $\underline{p}_{\phi}$, they comply with:

$$
\phi \underline{p_{\phi}}=\alpha K^{0} \cdot \Gamma^{0}(\underline{x}) *\left(K(\underline{x}) \cdot \delta K^{-1}(\underline{x}) \cdot \underline{p}_{\phi}\right)+\beta \Delta^{0}(\underline{x}) \cdot\left(\delta K^{-1}(\underline{x}) \cdot \underline{p}_{\phi}\right)
$$

The average of $\underline{p}_{\phi}$ over the volume of the unit cell being zero, $\underline{p}_{\phi}$ complies with:

$$
\underline{p}_{\phi}=\left(\Delta^{0}(\underline{x}) \cdot R^{0}+K^{0} \cdot \Gamma^{0}(\underline{x})\right)^{*} \underline{\phi}_{\phi}
$$

It follows that equation (27) can be put into the form:

$$
\boldsymbol{K}^{0} \cdot \Gamma^{0}(\underline{x}) * \underline{j}_{\phi}+\Delta^{0}(\underline{x}) * \underline{e}_{\phi}=0
$$

with:

$$
\underline{j}_{\phi}=\alpha K(\underline{x}) \cdot \delta K^{-1}(\underline{x}) \cdot \underline{p}_{\phi}-\phi \underline{\underline{p}_{\phi}} \quad \underline{e}_{\phi}=\beta \delta K^{-1}(\underline{x}) \cdot \underline{p}_{\phi}-\phi R^{0} \cdot \underline{p}_{\phi}
$$

Owing to property equation (29), $e_{\phi}$ and $j_{\phi}$ are rotational and divergence free, respectively. Indeed, applying the operator $\mathbf{P}$ to equation (29) leads, in Fourier space, to $\mathrm{P}(\underline{\xi}) \cdot \mathbf{K}^{0}(\underline{\xi}) \Gamma^{0}(\underline{\xi}) \cdot \underline{j}_{\phi}(\underline{\xi})=0$. But, from the definition of $\Gamma^{0}$, one obtains $\mathrm{P}(\underline{\xi}) \cdot \mathbf{K}^{0}(\underline{\xi}) \cdot \Gamma^{0}(\underline{\xi})=\mathrm{P}(\underline{\xi})$, which, from the properties of $\mathrm{P}$, shows that $j_{\phi}$ is divergence free. In the same way, applying the operator $\mathbf{Q} \cdot \mathbf{R}^{0}$ to equation (29) produces $\mathrm{Q}^{*} \mathrm{e}_{\phi}=0$, showing that $\underline{e}_{\phi}$ is rotational free. Finally, $\underline{e}_{\phi}$ and $j_{\phi}$ constitute the solutions of a problem related to a composite having the conductivity $\vec{K}^{\prime}(\underline{x})$ defined by:

$$
K^{\prime}(\underline{x})=\left[\alpha K(\underline{x}) \cdot \delta K^{-1}(\underline{x})-\phi I\right] \cdot\left[\beta \delta K^{-1}(\underline{x})-\phi R^{0}\right]^{-1}
$$


and subjected to a zero polarization. That problem has the trivial solution $\underline{p}_{\phi}=0$ if $K^{\prime}(\underline{x})$ is strictly positive or negative definite. A necessary condition for having the convergence of the iterativescheme is that the previously defined boundary problem has no solution when $\phi$ is outside [0,2[. This can be achieved if tensor $\boldsymbol{K}^{\prime}(\underline{x})$ is strictly positive or negative definite when $\phi \in]-\infty, 0[$ and $\phi \in[2,+\infty$ [. Considering the isotropic case, one has $\boldsymbol{K}^{\prime}(\underline{x})=k^{\prime}(\underline{x}) \boldsymbol{I}$ where $k^{\prime}(\underline{x})$ is given by:

$$
k^{\prime}(\underline{x})=k_{0} \frac{\alpha k(\underline{x})-\phi\left(k(\underline{x})-k_{0}\right)}{\beta k_{0}-\phi\left(k(\underline{x})-k_{0}\right)}
$$

It can be verified that, if $\alpha, \beta, k_{0}$ comply with:

$$
0 \leq \alpha<2, \quad-2<\beta \leq 0, \quad-\infty<h_{0}<0
$$

$k^{\prime}(\underline{x})$ is strictly positive and negative when $\phi<0$ and $\phi \geq 2$ and the convergence is ensured. Note that $k_{0}$, which was at first considered as the conductivity of the reference medium, is now negative. It is worth noticing that the above conditions are independent of the conductivity of the phases of the composite. It is then expected that the convergence of the PBIS equation (24) is obtained independently of the local properties of the composite. This will be confirmed through numerical calculations.

\subsection{Numerical evaluation of the rate of convergence}

We aim now at demonstrating the capacity of the new iterative scheme. We first consider the example shown in Figure 1. We represent the number of iterations at convergence as a function of $\log \left(k_{I} / k_{M}\right)$ in Figure 4. It is recalled that $k_{I}$ and $k_{M}$

Figure 4.

Diagram of the number of iterations at convergence of the PBIS as a function of the ratio $k_{I} / k_{M}$

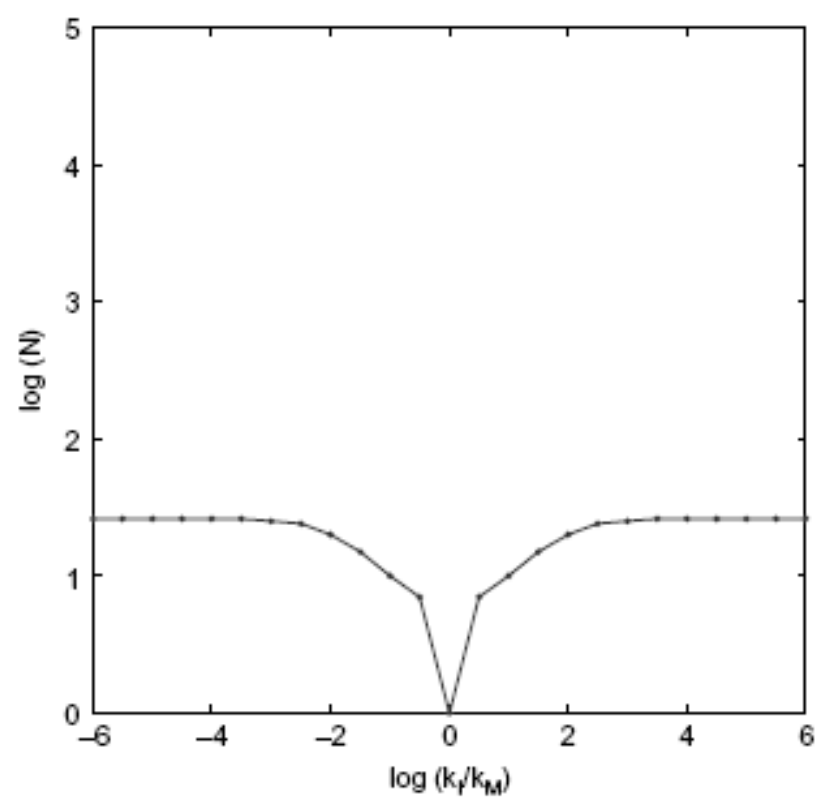


represent, respectively, the conductivity of the inclusion and of the matrix. It can be observed that for all values of the ratio $k_{I} / k_{M}$ the convergence of the algorithm is obtained quickly. The conditions given by equation (33) ensure the convergence of the iterative scheme but do not deliver the optimal values for $\alpha, \beta$ and $k_{0}$, i.e. leading to the lowest number of iterations at the convergence. In the absence of theoretical arguments yielding to the optimal choice of $\alpha, \beta$ and $k_{0}$, they must be determined numerically. From our numerical tests, the following values have been chosen: $\alpha=3 / 2, \beta=-3 / 2$ and $k_{0}=-k_{M}$ since they appear to be optimal for this numerical scheme. These values has been also considered for the other applications proposed in this paragraph.

To check the ability of this new approach, we consider a second example corresponding to a three phase composite. In this example a matrix with the conductivity $k_{M}$ contains two kinds of inclusions. The first one has the conductivity $k_{I a}=c k_{M}$ (gray inclusions on Figure 5), the second one has conductivity $k_{I b}=k_{M} / c$ (black inclusions on Figure 5).

The Figure 6 represents the number of iterations at convergence for the three iterative schemes: the PIS, the dual iterative scheme (DIS) and the PBIS. The logarithm of the number of iterations increases linearly with $\log (c)$ for both basic schemes. As expected, the case of a composite with zero and infinite conductivity inclusion (corresponding to $c=+\infty$ ) cannot be handled by these two methods. When the PBIS is used, the number of iterations increases with $c$ but reaches moderate values. Indeed, for higher values of the contrast $c$, the number of iterations increases up to $N=26$ iterations. When the PIS is used, the conductivity $k_{0}=\left(k_{\min }+k_{\max }\right) / 2=$ $k_{M}(c+1 / c) / 2$ has been used. For the DIS the resistivity of the reference medium is chosen as follows: $1 / k_{0}=\left(1 / k_{\min }+1 / k_{\max }\right) / 2=(c+1 / c) /\left(2 k_{M}\right)$.

For completeness, we consider the case of the diamond inclusion considered by Brisard and Dormieux (2010). The diamond reinforced composite is shown on Figure 7. It consists in aligned cylindrical inclusion having a square cross-section and rotated by an angle $\pi / 4$. The width of the squares is $a / \sqrt{2}$ and the distance between two neighboring inclusions is $2 b$. In our applications we have chosen $a=b / 2$.

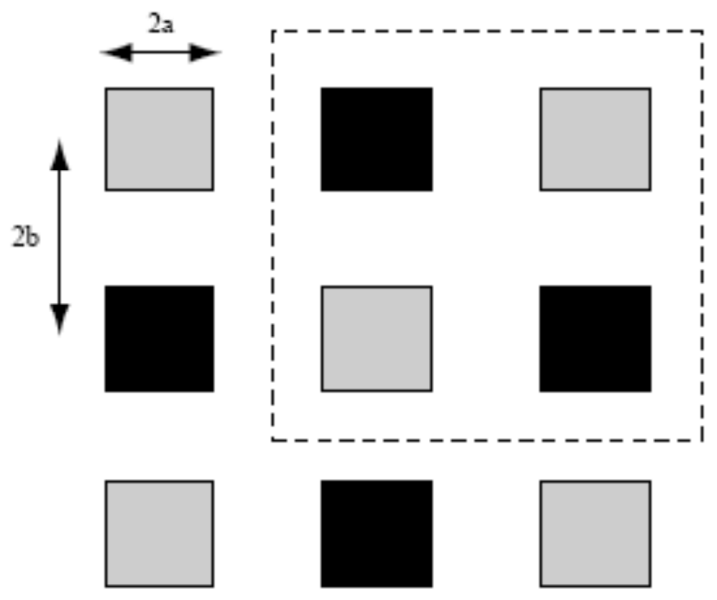




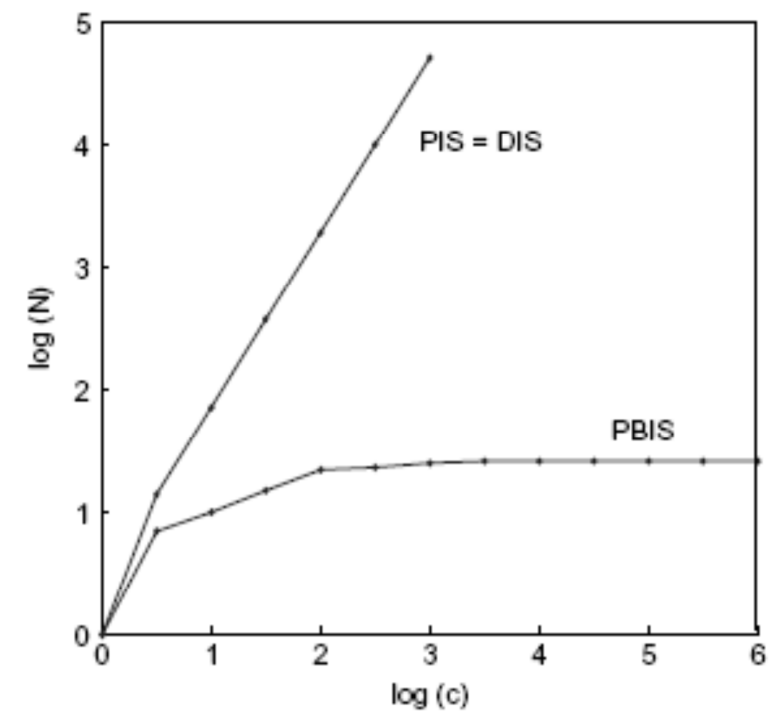

Figure 6.

Diagram of the number of iterations at convergence of the PBIS as a function of the ratio $k_{I} / k_{M}$

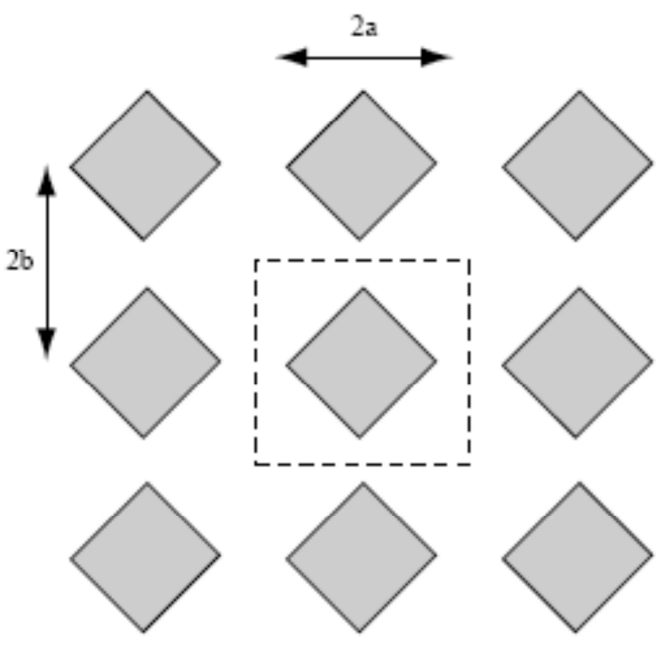

Figure 7.

The unit cell of the periodic composite

For this geometry of inclusions, the shape functions are defined by:

$$
\begin{aligned}
& \forall \underline{\xi} \neq 0:\left\{\begin{array}{l}
I_{I}(\xi)=\frac{\cos \left(a \xi_{2}\right)-\cos \left(a \xi_{1}\right)}{b^{2}\left(\xi_{1}^{1}-\xi_{2}^{2}\right)} \\
I_{M}(\xi)=-I_{I}(\underline{\xi})
\end{array}\right. \\
& \forall \underline{\xi}=0: \quad I_{I}(\xi)=\frac{a^{2}}{2 b^{2}}, \quad I_{M}(\xi)=1-\frac{a^{2}}{2 b^{2}}
\end{aligned}
$$


On Figure 8 we provide the number of iterations as a function of the contrast $k_{I} / k_{M}$ obtained with the PIS (the full line with points), the DIS (the full line with stars) and the polarization-based approach (the full line with circles). It can be noted that the results obtained with the diamond inclusions are similar with the ones already provided for square reinforced composites. The polarization approach converges quickly whatever the value of the contrast. For lowly conductive inclusions, the convergence rate of the PBIS coincides with the one of the PIS but coincides with the one of the dual approach when the case of lowly resistive inclusions is considered.

\section{Conclusion}

Anew Fourier based method has been proposed for computing theeffectiveproperties of composite conductors. The approach uses an iterative scheme for solving a problem in which a uniform polarization is prescribed over the unit cell instead of a uniform gradient of temperature or flux in the classical approaches. The method uses the same ingredients as the basic schemes: the periodic Green tensors, the FFT algorithm for computing the Fourier transform and its inverse. It has been shown that the conditions ensuring the convergence of this new iterativescheme areindependent of the value of the conductivities of the phases constituting the heterogeneous material. This result has been proven theoretically and tested numerically through several examples. More specifically, it has been shown that the problem of composites containing simultaneously phases having zero and infinite conductivity can be solved by this

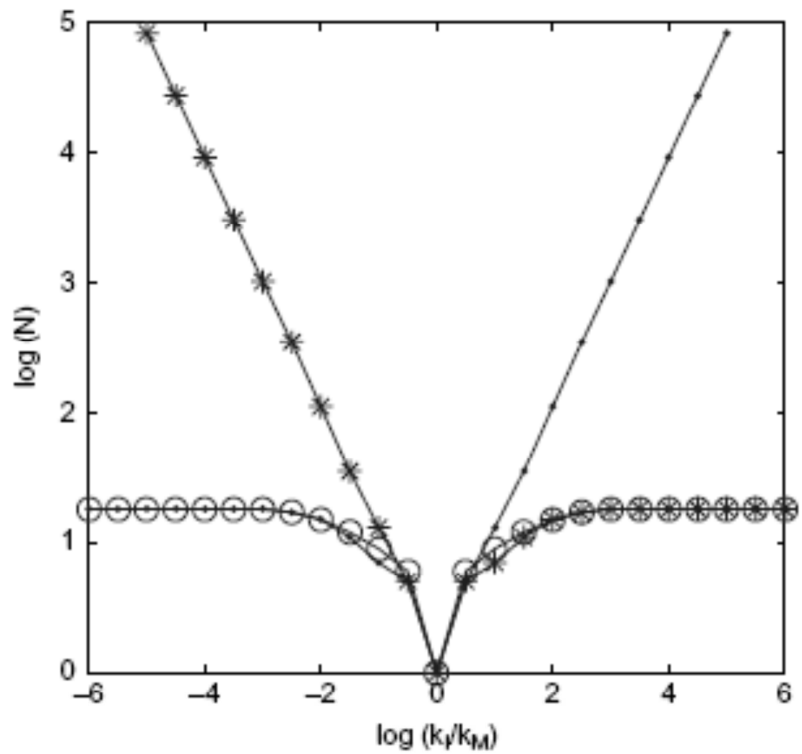

Notes: The case of the diamond inclusions; comparisons between the three iterative schemes: the PIS (the full line with points), the DIS (the full line with stars) and the polarization based approach (the full line with circles)
Figure 8.

Diagram of the number of iterations at convergence of the PBLS as a function of the ratio $k_{I} / k_{M}$ 
method with a very good rate of convergence while the resolutions with basic schemes lead to the divergence. The method can be extended to other various physical phenomena such as elasticity, piezoelectricity, etc.

\section{References}

Bhattacharya, K. and Suquet, P.M. (2005), "A model problem concerning recoverable strains of shape-memory polycrystals", Proc. Roy. Soc. London A, pp. 2797-2816.

Bonnet, G. (2007), "Effective properties of elastic periodic composite media with fibers", J. Mech. Phys. Solids, pp. 881-899.

Brenner, R. (2009), "Numerical computation of the response of piezoelectric composites using Fourier transform", Phys. Rev. B, p. 184106.

Brisard, S. and Dormieux, L. (2010), "FFT-based methods for the mechanies of composites: a general variational framework", Comput. Mater. Science, №. 3, pp. 663-671.

Brown, W. (1955), "Solid mixture permittivities", J. Chem. Phys, pp. 1514-1517.

Eischen, J.W. and Torquato, S. (1993), "Determining elastic behavior of composites by boundary element method", J. Appl. Phys, pp. 159-170.

Eyre, D.J. and Milton, G.W. (1999), "A fast numerical scheme for computing the response of composites using grid refinement", J. Phys, Vol. III, pp. 41-47.

Greengard, L. and Moura, M. (1994), "On the numerical evaluation of electrostatic fields in composite materials", Acta Numerica., pp. 379-410.

Gusev, A. (1997), "Representative volume element size for elastic composites: a numerical study", J. Mech. Phys. Solds, No. 7, pp. 1449-1459.

Hashin, Z. and Shtrikman, S. (1962), "On some variational principles in anisotropic and nonhomogeneous elasticity", J. Mech. Phys. Sol, No. 4, pp. 335-342.

Helsing. J. (1995), "An integral equation method for elastostatics of periodic composites", J. Mech. Phys. Solids, No. 6, pp. 815-828.

Kim, IC. and Torquato, S. (1962), "Effective conductivity of composites containing spherodal inclusions: comparison of simulations with theory", J. Appl. Phy., No. 10, pp. 3125-3131.

Kroener, E. (1972), Statistical Continuum Mechanics, Springer, Wien.

Lu, S.Y. and Lin, H.C. (1996), "Effective conductivity of composites containing aligned spheroidal inclusions of finite conductivity", J. Appl. Phys, No. 9, pp. 6761-6769.

McPhedran, R.C. and Movchan, A.B. (1994), "The Rayleigh multipole method for linear elasticity", J. Mech. Phys. Solids, pp. 711-727.

Mercier, S., Molinari, A. and El Mouden, M. (2000), "Thermal conductivity of composite material with coated inclusions: applications to tetragonal array of spheroids", J. Appl. Phys, №. 7, pp. 3511-3519.

Michel, J.C., Mbulinec, H. and Suquet, P. (2000), "A computational method based on augmented lagrangians and fast Fourier transforms for composites with high contrast", Comput. Model. Eng. Sci, No. 2, pp. 9-88.

Michel, J.C., Moulinec, H. and Suquet,P. (2001), "A computational scheme for linear and non-linear composites with arbitrary phase contrast", Int. J. Numer. Meth. Engng., pp. 139-160.

Milton, G.W. (2002), The Theory of Composites, Cambridge University Press, Cambridge.

Moulinec, H. and Suquet, P. (1994), "A fast numerical method for computing the linear and nonlinear mechanical properties of composites", C.R. Acad. Sci, No. 11, pp. 1417-1423. 
Myroshnychenko, V. and Brosseau, C. (2005), "Finite-element method for calculation of the effective permittivity of random inhomogeneous media", Phys. Rev. E, p. 016701.

Willis, J.R. (1977), "Bounds and self-consistent estimates for the overall properties of anisotropic composites", J. Mech. Phys. Solids, Vol. 25 No. 3, pp. 185-202.

Appendix 1. The convergence of the primal iterative scheme

We first investigate the convergence of the primal iterative scheme. For simplicity, our analysis is restricted to the case of isotropy, then we put $K(\underline{x})=k(\underline{x}) I$ and $K^{0}=k_{0} I$. The convergence of the iterativescheme (15) is ensured if all the eigenvalues of theoperator $I-\Gamma^{0}(\underline{x}) * K(\underline{x})$, are comprised in the interval ] - 1,1] or, equivalently, the eigenvalues of $\Gamma^{0}(x)^{*} K(x)$, are comprised in the interval [0,2[. By $\phi$ we define an eigenvalue and $\epsilon_{\phi}$ an associated eigenvector of this operator:

$$
\phi \underline{e}_{\phi}=\left(\Gamma^{0}(\underline{x}) * K(\underline{x})\right) \cdot \underline{e}_{\phi}
$$

Due to the property of the Green's tensor $\Gamma^{\circ}(\underline{x})$ (Section 2$), \underline{\varepsilon}_{\phi}$ is rotational free and has a null volume average. Such field complies with: $\underline{\underline{e}}_{\phi}=\Gamma^{0}(\underline{x})^{*}\left[K^{0}: \underline{e_{\phi}}\right]$. Equation (A1) can then be rewritten as:

$$
\overrightarrow{\Gamma^{0}}(\underline{x})^{*}\left[K(\underline{x})-\phi K^{0}\right] \cdot \underline{e}_{\phi}=0
$$

Which indicates that $K^{\prime}(\underline{x}) \cdot \underline{e}_{\phi}$, where $K^{\prime}(\underline{x})=K(\underline{x})-\phi K^{0}$, is a divergence free vector. Then, $\underline{e}_{\phi}$ can beinterpreted as gradient of temperature, solution of a problem of composite with the conductivity $K^{\prime}(\underline{x})$ and subjected toa zerouniform gradient of temperature. Such a problem has the trivial solution $\ell_{\phi}=0$ if $K^{\prime}(x)$ is positive or negative definite. A sufficient condition for having the convergence is that $k^{\prime}(\underline{x})=k(\underline{x})-\phi b_{0}$ is strictly positive or negative definitewhen $\phi<0$ and $\phi \geq 2$. In thisway, all the eigenvalues will be comprised in the interval [0,2 [. At this stage it is easy to recover equation (17).

Appendix 2. The convergence of the dual iterative scheme

The same procedure is employed for obtaining the conditions of convergence of the dual iterative scheme (19). We introduce $\phi$ and $j_{\phi}$ an eigenvalue and an associated eigenvector of $\Delta^{0}(\underline{x})^{*} R(\underline{x})$ :

$$
\phi \underline{j}_{\phi}=\left(\Delta^{0}(\underline{x})^{*} R(\underline{x})\right) \cdot \underline{e_{\phi}}
$$

We seardh the conditions for which all the eigenvahues $\phi$ are comprised in $\left[0,2\left[\right.\right.$. In (B1), $j_{\phi}$ is a divergence free vector which has a zero volume average, it complies with $j_{\phi}=\Delta^{0}(\underline{x})^{*}\left[R^{0} \cdot \underline{j}_{\phi}\right]$ and:

$$
\Delta^{0}(\underline{x})^{*}\left(R(\underline{x})-\phi R^{0}\right) \cdot \underline{j}_{\phi}=0
$$

Which indicates that $R^{*}(\underline{x}) \cdot j_{\phi}$, with $R^{*}(\underline{x})=R(\underline{x})-\phi R^{0}$, is a rotational free vector.Consequently, $j_{\phi}$ can be interpreded as a flux, sohution of a problem of a composite with the resistivity $R^{*}(\underline{x})$ and subjected to a zero uniform flux. Again we search the condition for which that problem has the trivial solution $j_{\phi}=0$ when $\phi<0$ and $\phi \geq 2$. For an isotropic composite, the resistivity is $1 / k^{*}(\underline{x})=1 / k(\underline{x})-\phi / k_{0}$. It can be shown that $1 / k^{*}(\underline{x})$ is strictly positive or negative definite for $\phi<0$ and $\phi \geq 2$ if the condition (21) is ensured.

\section{Corresponding author}

Vincent Monchiet can be contacted at: vincent.monchiet@univ-paris-est.fr 